\title{
TOEPLITZ OPERATORS IN A QUARTER-PLANE ${ }^{1}$
}

\author{
BY GILBERT STRANG
}

Communicated by Peter D. Lax, July 20, 1970

The key result about Toeplitz operators on a quarter-plane is apparently this, that the Fredholm alternative holds if the symbol $t$, in the class of nonvanishing functions on the torus, is homotopic to a constant. We want to give a simple proof.

Recall that the Toeplitz operator $T$ with symbol $t(\theta)=\sum t_{k} e^{-i k \theta}$, acting on a vector $v$, is defined by

$$
(T v)_{i}=\sum_{j \geq 0} t_{i-j} v_{j}, \quad i \geqq 0 .
$$

In the classical one-dimensional case, $i, j$, and $k$ are integers, $\theta$ is a scalar, and $T$ is represented by the discrete convolution matrix

$$
\left(\begin{array}{lll}
t_{0} & t_{1} & t_{2} \\
t_{-1} & t_{0} & t_{1} \\
t_{-2} & t_{-1} & t_{0} \\
\ldots & \ldots
\end{array}\right) .
$$

On the quarter-plane, $i$ and $j$ are pairs of nonnegative integers, $\theta=\left(\theta_{1}, \theta_{2}\right), k \theta=k_{1} \theta_{1}+k_{2} \theta_{2}$, and $T$ can be represented by an array (2) in which each entry is itself a Toeplitz operator on a half-line-or equivalently, by a four-dimensional analogue of (2). We assume $\sum\left|t_{k}\right|<\infty$, so that $T$ is a bounded operator on any of the spaces $l_{+}^{p}$, normed by

$$
\|v\|_{p}=\left(\sum_{j \geq 0}\left|v_{j}\right|^{p}\right)^{1 / p} .
$$

After Fourier transform, taking the vector $v$ into $v(\theta)=\sum v_{j} e^{i j \theta}$, the action of $T$ remains easy to describe:

$$
(T v)(\theta)=P(t(\theta) v(\theta)),
$$

where $P$ is projection onto the nonnegative frequencies,

$$
P \sum a_{j} e^{i j \theta}=\sum_{j \geq 0} a_{j} e^{i j \theta} .
$$

AMS 1969 subject classifications. Primary 4255, 4725; Secondary 4425.

Key words and phrases. Toeplitz operator, Wiener-Hopf factorization, convolution, Fredholm alternative, index theorem.

1 This research was supported by the National Science Foundation (GP-13778). 
In one dimension the complementary projection $P_{-}=I-P$ maps onto the negative frequencies $j<0$; in the plane there are projections $P_{2}=P_{-+}, P_{3}=P_{-}, P_{4}=P_{+-}$corresponding in an obvious way to the remaining three quadrants, with $P+P_{2}+P_{3}+P_{4}=I$.

Toeplitz operators on a half-line led to the first, and perhaps still the simplest, example of an index theorem: if $t$ is nonvanishing then $T$ is Fredholm, and its analytical index-the dimension of the kernel minus that of the cokernel-is equal to its topological index-the negative of the winding number around the origin of the curve traced by $t(\theta)$. In the quarter-plane (or in $R_{+}^{n}$ ) such an index theorem presents a challenge. The obvious topological index is the pair of integers corresponding to the homotopy class of $t$, i.e., the winding numbers of $t\left(\theta_{1}, 0\right)$ and $t\left(0, \theta_{2}\right)$. The proper analytical index is not so evident. (We remark that $T$ is not even Fredholm unless its index is $(0,0)$; for example, the shift symbolized by $t(\theta)=\exp \left(-i \theta_{1}\right)$ annihilates all $v$ of the form $v(\theta)=\sum v_{0 j} \exp \left(i j \theta_{2}\right)$, and thus $\operatorname{dim}(\operatorname{ker} T)=\infty$.) Perhaps the index specifies the number of conditions at the boundaries of the quarter-plane required to make $T$ Fredholm.

We want to study only the case in which the Fredholm alternative holds - the range of $T$ is closed, and $\operatorname{dim}(\operatorname{ker} T)=\operatorname{dim}(\operatorname{coker} T)$. On the half-line, according to the index theorem, this occurs if and only if $t$ has winding number zero-that is, when $\arg t(2 \pi)=\arg t(0)$, so that $t$ is homotopic to a constant. In this case $t$ admits a Wiener-Hopf factorization into $t_{-} t_{+}$, where

$$
t_{-}=\exp \left(P_{-} \log t(\theta)\right), \quad t_{+}=\exp (P \log t(\theta)),
$$

and more than just the Fredholm alternative is true: $T$ is actually $i n$ vertible. In fact, $T^{-1}$ is given explicitly by $\left(t_{+}(\theta)\right)^{-1} P\left(t_{-}(\theta)\right)^{-1}$.

It would be wonderful if such a trick worked also for the quarterplane problem; apparently, it does not. It is still possible and useful, if the symbol is homotopic to a constant, to factor it into

$$
t=t_{1} t_{2} t_{3} t_{4}, \quad \text { where } t_{i}=\exp \left(P_{i} \log t\right), P_{1} \equiv P .
$$

As Krein has observed, the Wiener-Lévy theorem assures that $\log t$ (and therefore each $t_{i}$ and $t_{i}^{-1}$ ) has an absolutely convergent Fourier series, as $t$ had. This factorization again yields explicit inverses to the two half-plane operators, acting on the ranges of $\left(P+P_{2}\right)$ and $\left(P+P_{4}\right)$, respectively; cf. Goldenstein and Gohberg [1], [2]. These operators and their inverses are given by

$$
\begin{array}{ll}
T_{U v}=\left(P+P_{2}\right) t v, & T_{U}^{-1} w=t_{1}^{-1} t_{2}^{-1}\left(P+P_{2}\right) t_{3}^{-1} t_{4}^{-1} w, \\
T_{R} v=\left(P+P_{4}\right) t v, & T_{R}^{-1} w=t_{1}^{-1} t_{4}^{-1}\left(P+P_{4}\right) t_{2}^{-1} t_{3}^{-1} w .
\end{array}
$$


We verify, for example, that

$$
\begin{aligned}
& t_{1}^{-1} t_{2}^{-1}\left(P+P_{2}\right) t_{8}^{-1} t_{4}^{-1}\left(P+P_{2}\right) t v \\
& \quad=t_{1}^{-1} t_{2}^{-1}\left(P+P_{2}\right) t_{1} t_{2} v-t_{1}^{-1} t_{2}^{-1}\left(P+P_{2}\right) t_{3}^{-1} t_{4}^{-1}\left(P_{3}+P_{4}\right) t v=v,
\end{aligned}
$$

since $\left(P+P_{2}\right)$ acts in the first term on an element in its range, and in the second term on an element in its null-space.

On the quarter-plane, however, we have to face the possibility that even though the symbol $t$ is homotopic to a constant, the Toeplitz operator T may fail to be invertible. A class of simple and very elegant examples has been given by Douglas and Howe [3], refuting the proof of invertibility claimed by Malyšev [4]. (We want to call the reader's attention to the paper [3], which begins the development of a fullscale theory of Toeplitz operators in $R_{+}^{u}$, and contains an independent proof both of the theorem established below and of its converse.) There do exist additional properties of $t$ which guarantee the invertibility of $T$ : Malyšev's proof works for trigonometric polynomials $t$ of first degree, Osher [5] admits $t=p\left(\theta_{1}\right) q\left(\theta_{2}\right)+r\left(\theta_{2}\right)$, and-the simplest and most important class in practice - Busenberg [6] has extended the work of Kraut and Lehman [7] to prove invertibility if $t(\theta)$ stays in an open half-plane, say $\operatorname{Re} t>0$ for all $\theta$. The Douglas-Howe examples show, on the other hand, that in general invertibility will remain extremely elusive, and the following result is perhaps the more natural one:

THEOREM. If $t\left(\theta_{1}, \theta_{2}\right)$ is homotopic to $t \equiv 1$ in the class of nonvanishing symbols, then the Fredholm alternative holds for $T$. Furthermore, the operator $Q=P\left(T_{U}^{-1}+T_{R}^{-1}-t^{-1}\right) P$ is an explicit quasi-inverse for $T: Q T$ and $T Q$ differ from the identity by a compact operator.

REMARK. The force of this last statement is that although the Wiener-Hopf factorization fails to lead precisely to $T^{-1}$, as it was able to do on the half-line, still it misses only by a compact operator. In fact, the reason $Q$ succeeds this well is that each of the half-plane inverses gets one boundary right and one wrong, while multiplication by $t^{-1}$ on the whole plane gets them both wrong, and cancels just as needed, modulo a compact operator.

Proof. We shall verify explicitly that $Q$ is a quasi-inverse, implying that $T$ is Fredholm. Then to show that the Fredholm alternative holds, i.e., that index $(T)=0$, we use the invariance of the index under homotopy: the homotopy which connects the given $t$ to the symbol $t \equiv 1$ induces an operator homotopy, connecting $T$ to the identity operator, and its index is obviously zero. This technique was applied 
by Lax [8] to determine the index of block-Toeplitz operators on the half-line; Nirenberg proposed $P t^{-1} P$ as quasi-inverse in that problem.

Now our only task is to verify that

$$
\begin{aligned}
& P\left(T_{U}^{-1}+T_{R}^{-1}-t^{-1}\right) P t P=P+\text { compact, } \\
& P t P\left(T_{U}^{-1}+T_{R}^{-1}-t^{-1}\right) P=P+\text { compact. }
\end{aligned}
$$

It is convenient to regard all these operators as acting on "the whole plane", i.e., on $l^{p}\left(Z^{2}\right)$; recall that $P$ is projection with range $l_{+}^{p}=l^{p}\left(Z_{+}^{2}\right)$. We start with

$$
\begin{aligned}
& P T_{U}^{-1} P t P=P T_{U}^{-1}\left(P+P_{2}\right) t P-P T_{U}^{-1} P_{2} t P \\
& =P-P T_{U}^{-1} P_{2} t P \quad \text { by (7). }
\end{aligned}
$$

The last operator is not compact; it is the error at the left boundary of the quarter-plane. Similarly,

$$
\begin{gathered}
P T_{R}^{-1} P t P=P-P T_{R}^{-1} P_{4} t P, \\
-P t^{-1} P t P=-P+P t^{-1} P_{2} t P+P t^{-1} P_{4} t P+P t^{-1} P_{3} t P .
\end{gathered}
$$

In adding these three equations, we need to combine

$$
\begin{aligned}
& -P T_{U}^{-1} P_{2} t P+P t^{-1} P_{2} t P=P t_{1}^{-1} t_{2}^{-1}\left(P_{3}+P_{4}\right) t_{3}^{-1} t_{4}^{-1} P_{2} t P, \\
& -P T_{R}^{-1} P_{4} t P+P t^{-1} P_{4} t P=P t_{1}^{-1} t_{4}^{-1}\left(P_{3}+P_{2}\right) t_{2}^{-1} t_{3}^{-1} P_{4} t P .
\end{aligned}
$$

Now the sum we want is

$$
\text { (8) }=(10)+(11)+(12)=P+P t^{-1} P_{3} t P+(13)+(14) \text {, }
$$

and we have to show that these last three terms represent compact operators. To do so, we use an observation made independently (and much earlier) by Simonenko [9]. (We note that Malyšev credits Simonenko with the first proof of the present theorem; Simonenko treated continuous convolutions-Wiener-Hopf integral operators, to which our arguments also apply - and Douglas and Howe show that his conclusions lead to the unexpected result that those operators are not only Fredholm but invertible.) The observation itself is very simple and we omit its proof.

Lemma. If $P_{\boldsymbol{i}}$ and $P_{j}$ are projections onto diagonally opposite quadrants, and the symbol $\tau$ has absolutely convergent Fourier series, then $P_{i} \tau P_{j}$ is a compact operator on $l^{p}\left(Z^{2}\right)$.

The application to $P t^{-1} P_{3} t P$, and to (13) and (14), is immediate; 
each term contains such a product $P_{i} \tau P_{j}$, and is therefore compact. This verifies (8) and, together with a parallel verification of (9), proves the theorem.

Finally we mention the block-Toeplitz case, in which the symbol $t$ is a matrix function. Assuming the half-plane operators are invertible, our operator $Q$ still provides a quasi-inverse, so that $T$ is once again Fredholm. (The factorization of $t$ is no longer given by (5).) However, it is not clear how to show that $t$ is homotopic to the identity matrix, while keeping both half-plane operators invertible along the way. Therefore, we are unable to conclude that the index of $T$ is zero.

\section{REFERENCES}

1. L. S. Gol'denšteln and I. C. Gohberg, On a multi-dimensional integral equation on a half-space whose kernel is a function of the arguments and on a discrete analogue of this equation, Dokl. Akad. Nauk SSSR 131 (1960), 9-12=Soviet Math. Dokl. 1 (1960), 173-176. MR 22 \#8298.

2. L. S. Gol'denšteIn, Criteria for one-sided invertibility of functions of several isometric operators and their applications, Dokl. Akad. Nauk SSSR 155 (1964), 28-31 =Soviet Math. Dokl. 5 (1964), 330-334. MR 28 \#4403.

3. R. G. Douglas and R. Howe, On the $C^{*}$-algebra of Toeplitz operators on the quarter-plane (to appear).

4. V. A. Malyšev, On the solution of discrete Wiener-Hopf equations in a quarterplane, Dokl. Akad. Nauk SSSR 187 (1969), 1243-1246=Soviet Math. Dokl. 10 (1969), 1032-1036.

5. S. J. Osher, On certain Toeplitz operators in two variables, Pacific J. Math. (to appear).

6. S. N. Busenberg, Iterative solution of the Wiener-Hopf problem in several variables, Proc. Amer. Math. Soc. (submitted).

7. E. A. Kraut and G. W. Lehman, Diffraction of electromagnetic waves by a rightangle dielectric wedge, J. Mathematical Phys. 10 (1969), 1340-1348.

8. P. D. Lax, Toeplitz operators, AFOSR Lectures in Differential Equations, George Washington University, Washington, D. C., 1967.

9. I. B. Simonenko, Operators of convolution type in cones, Mat. Sb. 74 (116) (1967) 298-313 = Math. USSR Sb. 3 (1967), 279-293. MR 36 \#5773.

Massachusetts Institute of Technology, Cambridge, Massachusetts 02139 